\title{
DISCUSSION
}

\section{Issues in the care of the dying}

\section{SK JINDAL.}

Professor and Head, Department of Pulmonary Medicine, Postgraduate Institute of Medical Education and Research, Chandigarh 160 012 INDIA e-mail: skjindal@ indiachest.org

There is rejuvenated interest within modern medicine in end-oflife issues including the care of terminally ill and dying patients. Technological advances in the last few decades have made us believe that death is an unnatural event and that life can be prolonged at will. This has resulted in the adoption of lifesupporting measures, which are sometimes antagonistic to the very dignity of life.

Death is an inevitable conclusion of life. The dignity of death therefore is as important as that of life. The fortunate few die without much suffering, but most people face either the debility of old age or an incurable and progressive illness.

In medical practice, life-prolonging treatments are those with the potential to postpone death from incurable and terminal illnesses. These conditions may include advanced cancers, organ failure and progressive, end-stage neurological illnesses. Advances in modern technology have made it possible to sustain life for days, weeks or sometimes years. These involve treatments like cardiopulmonary resuscitation, artificial respiration, cancer chemotherapy, dialysis, artificial nutrition, hydration and multiple drugs.

The literature is replete with opinions and judgments on medical cases involving legal interventions. Most of these are in the West though there have been a few instances in India (1). With the rapid expansion of techno-medicine promising the moon to everyone, people's demands to live longer have also increased. But the costs are tremendous and there are wide gaps in the availability of resources, expertise, human power, infrastructure, awareness and knowledge in providing lifeprolonging treatment. This is a huge burden on the shoulders of health professionals and policy makers.

There are several important issues in the care of terminally ill patients. The most critical is related to continuation or discontinuation of life-prolonging treatments. The subject incorporates many legal, financial and ethical questions. Does an individual possess the right to refuse treatment? Who else can decide on treatment options? Who is to bear the costs of life-prolonging treatment? To what extent is the medical team responsible for terminal care?

\section{The right to refuse}

A most contentious subject relates to the decision of patients to refuse life-prolonging treatment. The law generally gives adults the right to refuse treatment. However, one must often decide whether a dying adult is competent to decide or even communicate his or her decision.

Legally speaking, adults are presumed to be competent to make decisions unless there are reasons to suppose otherwise. The right to refuse treatment is firmly established in British medical practice standards (2); the right to refuse treatment was upheld even in a patient diagnosed as psychotic who refused amputation of his gangrenous foot (3). In the United States, the Supreme Court in the Cruzan case is one of many which asserted the principle that individuals have the constitutional right to refuse treatment even if this may result in the person's death (4). This right has been reiterated in several other judgements even where patients did not have life-threatening illnesses.

\section{Who decides?}

Another important issue in terminal care is the concept of 'advance directive'. Sick patients may leave written or verbal directives expressing their wishes regarding resuscitation and terminal care. A 'Do Not Resuscitate' directive by a terminally ill patient is not an uncommon practice in western medicine.

Most advance directives have legal sanction in the United States. The Patient Self-Determination Act (1990) mandates for hospitals in this country to inform patients of their right to refuse medical treatment and to make advance directives.

\section{The cost of terminal care}

Excessive economic burden is an issue, which concerns most patients and their families. It is when resources are limited and hopes are dwindling that money must be spent as never before. There are cultural issues as well. Some people hope to buy a miracle. Some spend out of love, as repayment of their debt to the dying, or out of duty. I have heard people complain of this burden but very few opt to discontinue. Everyone hopes that someone else will decide. Who else can decide? It is a very hard decision to make.

The problem intensifies as the care gets prolonged, costs multiply and/or the dying person's health does not improve. A patient with a chronic respiratory or neuromuscular disease may become dependent on artificial respiratory support, which cannot be withdrawn. A patient may survive an acute insult but ends up in a functionally dependent state. Not uncommonly, a patient may suffer from permanent brain damage. Most of these situations drain the resources of the family as well as of society. It is contrary to ethics to have an economically ruined family because of a patient's admission into the ICU, especially when the health outcome is also dubious or incomplete. 


\section{Medical decisions and palliative care}

Relief from pain and suffering is an important issue at the end of life. Drugs may help but only to a limited degree. Unfortunately, most drugs required for this purpose are not necessarily safe. These drugs may produce 'unintended harm' to the patient invoking anger or even legal liabilities against the doctors. For example, sedatives, administered to produce some sleep or give relief from restlessness, breathlessness and extreme anxiety, may depress one's respiration and other brain functions. The patient may lapse into a coma and never awaken. Such risks are unavoidable, and constitute the phenomenon of "double effect". There are different opinions on the question of whether one should adopt a risky option for palliation or let a person suffer. The humane approach would be to palliate but this is fraught with inherent dangers of abuse. Caregivers and relatives with selfish motives and criminal intentions may also encourage palliative drugs. Medical and nursing personnel may inadvertently or rarely even intentionally get involved into avoidable liability suits.

The withdrawal or with holding of treatment and supportive care for the underlying disease may also fall in the same category but there are several other related issues. General guidelines can enable decisions in individual cases. In no case can this decision be left to a single party. Terminal care should not be confused or identified with any kind of euthanasia, active, passive or assisted. It is merely the administration of palliative care with an acceptance of its potential risks.

\section{The final judgment - legal or medical?}

Even more basic is the core issue of medical judgement and the sanctity it has to provide life-prolonging treatment. Medical practitioners tend to look to the law for support of their actions. No citizen, and certainly not a doctor, can afford to ignore the law. But medical judgements cannot always be guided by legal justifications, especially since the law is either silent or ambiguous on most issues related to the prolongation of life.

Unfortunately, we have little discussion or information on this subject in India. Legal as well as medical opinions guiding the judgements and actions of doctors in end-of-life care are yet evolving in this country. It is therefore useful to look into examples elsewhere. Most western medical associations have clear guidelines for decision-making. The British Medical Association has comprehensive recommendations on several issues with respect to decision-making on 'withholding' or 'withdrawing' life-prolonging treatment (5). Of course the guidelines can only aid in the process of decision-making; they cannot form a protocol of action. Doctors are essentially guided in their actions by the primary goal of medicine -- to restore or maintain the patient's health.

The real dilemma is for the doctor looking after a patient who has left a 'do not resuscitate' or 'refusal to accept treatment' order. The competence of such an advance directive is always challengeable. On the other hand, providing treatment against the expressed wishes of a patient may also land the doctor in trouble. Medical associations therefore recommend that wherever genuine doubts exist about the validity of an advance refusal, the doctor should act in favour of giving emergency treatment, buying more time to decide in consultation with the family and colleagues.

The debate on the dilemmas faced by caring doctors is bound to continue. No final protocol can be made for doctors to act. They must follow the local cuitural and medical practices. Nonetheless, more people are likely to assert their right to refuse life-prolonging treatments. The core philosophy in terminal care remains "Exit with dignity."

\section{References}

1. Mathias Lina. The right to life or the right to die? The Hindu, May 1 , 2005. Available from URL:http://www.hindu.com $/ \mathrm{mag} / 2005 / 05 / 01 /$ stories/2005050100390300.htm (accessed on July 15, 2005)

2. Prolonging treatments: Good Practices in Decision Making. Available from URL: http://www.gmc-uk.org/standards/whwd.htm (accessed on July $15,2005)$

3. Re C [1994] 1 WLR 1990. Case referred to in: UK Clinical Ethics Network Policy on Consent and Refusal of Treatment. Available from URL:http:// www.ethics-network.org.uk/Ethics/econsent.htm (accessed on July 15, 2005)

4. The Right to Die. Available from URL: http://www.law.umkc.edu/faculty/ projects/ftrials/conlaw/righttodie.htm (accessed on July 15, 2005)

5. British Medical Association. Withholding and withdrawing life-prolonging medical treatment. London, BMJ Books, 1999. 